\title{
Characterization of Void-Dominated Ductile Failure in Pure Ta
}

\author{
Blythe G. Clark ${ }^{1}$, Joseph R. Michael², Bonnie B. McKenzie², Jay Carroll², Hojun Lim², Brad L. Boyce² \\ 1. Sandia National Laboratories, Physical, Chemical, and Nano Sciences Center, Albuquerque, NM. \\ 2. Sandia National Laboratories, Materials Science and Engineering Center, Albuquerque, NM
}

Prediction of materials reliability requires a physical understanding of the failure process. In the case of void-dominated ductile failure, this includes an understanding of the processes and dominant microstructural variables that lead to void nucleation, growth, and coalescence.

Mechanistic descriptions of void-dominated ductile failure are largely based on studies from several decades ago that relied on optical microscopy techniques [1] [2]. This talk will discuss how modern techniques, such as scanning electron microscopy (SEM), electron backscattered diffraction (EBSD), site-specific focused ion beam (FIB) sample preparation, and transmission electron microscopy (TEM), can enable new insight into the ductile failure process, particularly at the nano to microscale. Because this work is part of a collaborative effort to develop predictive computational models for failure of BCC metals, experiments are focused on characterizing the void-dominated ductile failure process in pure Ta.

In metal alloys the initiation of voids is primarily driven by decohesion at second-phase particles or inclusions [3][4][5]. However, in pure ductile metals where these particles and inclusions are not present, such as in the $99.9 \%$ Ta used in this study, the process is inherently different. Initial tensile deformation experiments and post-mortem fractography as noted in Figure 1 show that Ta exhibits significant ductility, but with a valley and ridge surface [6] instead of the classic hemispherical dimpling commonly observed in ductile metal alloys [7]. Through SEM analysis of the fracture surfaces, it was determined that the mating fracture surfaces were mirrored: valleys mated to valleys, and ridges mated to ridges. Thus the deformation was void dominated, but the mechanism of void initiation was unclear.

To further study the void initiation process in Ta and the progression of damage towards failure, interrupted tensile specimens as a function of percent remaining strength were prepared. By polishing the samples to their mid-plane as seen in Figure 2a, where triaxial stresses are highest and thus voids likely to be present, we studied the progression of void structures as a function of strain. Using EBSD analysis to characterize local misorientations in voided regions, we determined that voids in Ta are formed within grains, as opposed to at grain boundaries, along bands of high misorientation with respect to the tensile axis. Figure $2 \mathrm{~b}$ shows elongated, inclined [001] subgrains alternating with regions of [122] associated with voids in a Ta tensile bar elongated to $60 \%$ remaining strength. We hypothesize that the dislocation processes giving rise to localized regions of high misorientation within grains are promoting the collection of vacancies, thus inducing void nucleation. This hypothesis is being further explored through site-specific FIB sample preparation and TEM analysis of dislocation structures in deformed Ta samples. 
References:

[1] K.E. Puttick, Philosophical Magazine 4 (1959), p. 954-969.

[2] H.C. Rogers, Transactions of the Metallurgical Society of AIME 218 (1960), p. 498-506.

[3] A. Needleman, Journal of Applied Mechanics 54 (1987), p. 525-31.

[4] D. Broek, Engineering Fracture Mechanics 5 (1973), p. 55-66.

[5] A.S. Argon, J. Im, and R. Safoglu, Metallurgical Transactions A 6A (1975), p. 825-37.

[6] B. L. Boyce, B. G. Clark, P. Lu, J. D. Carroll, and C. Weinberger, Metallurgical and Materials Transactions A 44A (2013), p. 4567-4580.

[7] R. H. Van Stone, T. B. Cox, J. R. Low, Jr. and J. A. Psioda, International Materials Reviews 30 (1985), p. 157-180.

[8] The authors would like to thank Drs. Corbett Battaile and Khalid Hattar for useful discussions.

[9] Sandia National Laboratories is a multi-program laboratory managed and operated by Sandia Corporation, a wholly owned subsidiary of Lockheed Martin Corporation, for the U.S. Department of Energy's National Nuclear Security Administration under contract DE-AC04-94AL85000.

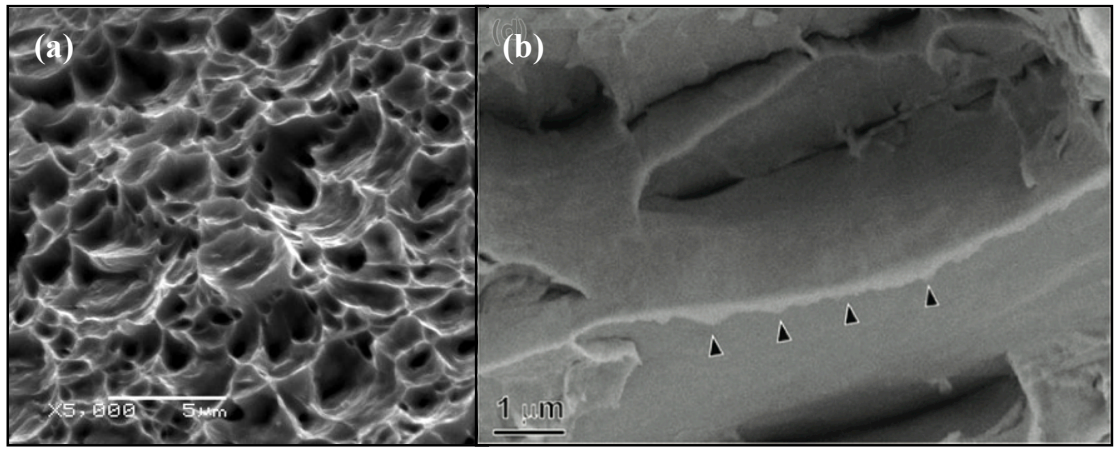

Figure 1. SEM images comparing (a) a ductile dimple fracture surface of 304L stainless steel, with (b) a valley and ridge fracture surface of $99.9 \%$ Ta. Both materials were deformed quasistatically at room temperature.
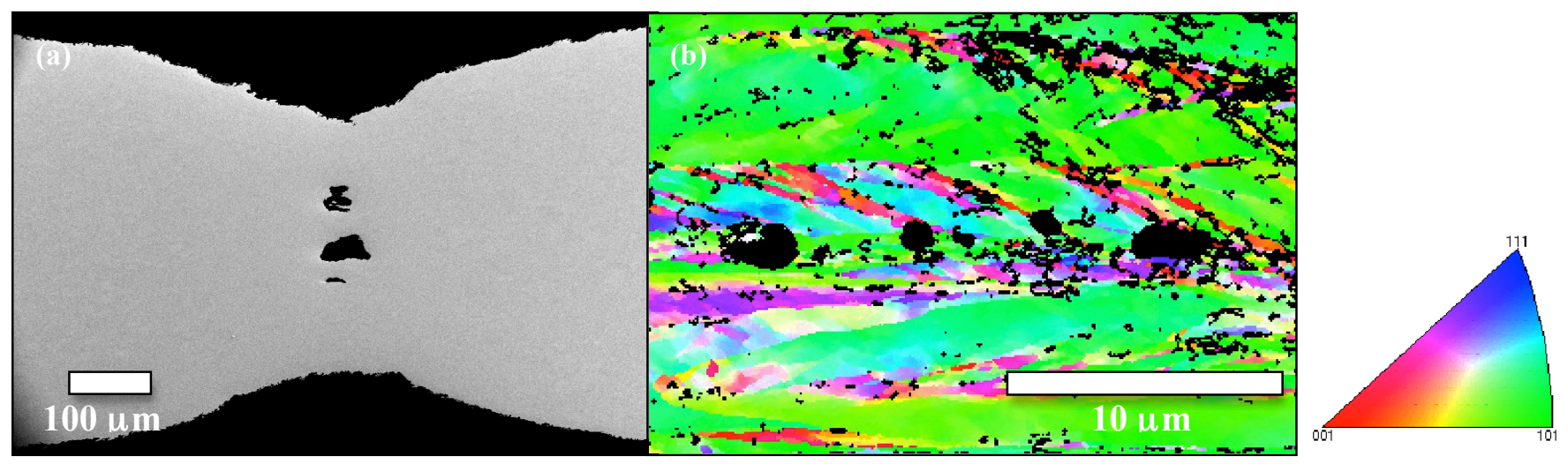

Figure 2. (a) Example of a Ta tensile bar deformed quasistatically to $40 \%$ remaining strength and polished to the mid-plane for void analysis. (b) EBSD data collected around a localized region of deformation-induced voids in a Ta tensile bar deformed quasistatically to $60 \%$ remaining strength. Orientation color key is given in the stereographic triangle. Here, orientations were plotted with respect to the tensile axis. 